\title{
CONGENITAL DIASTASIS OF TIBIO-FIBULAR MORTISE
}

\author{
S. M. Tuli and B. P. Varma, Varanasi, India \\ From the Department of Orthopaedic Surgery, Institute of Medical Sciences, \\ Banaras Hindu University, Varanasi
}

This paper describes what is thought to be a hitherto unreported condition of congenital diastasis of the inferior tibio-fibular mortise. The available English literature has been extensively reviewed, and no report of such a condition was found.

\section{CASE REPORTS}

Case 1 (S. M. T.'s case) - A five-months-old girl was seen in October 1969 with congenital club foot on the right side. She was born normally, the first child of apparently healthy parents. There was no known history of congenital anomalies in the family. The child had not received any treatment since birth. Examination revealed an equinovarus deformity of the right foot, with an unduly prominent and distally placed medial malleolus and a widened inferior tibio-fibular mortise as compared with the normal (Fig. 1). The right leg was two

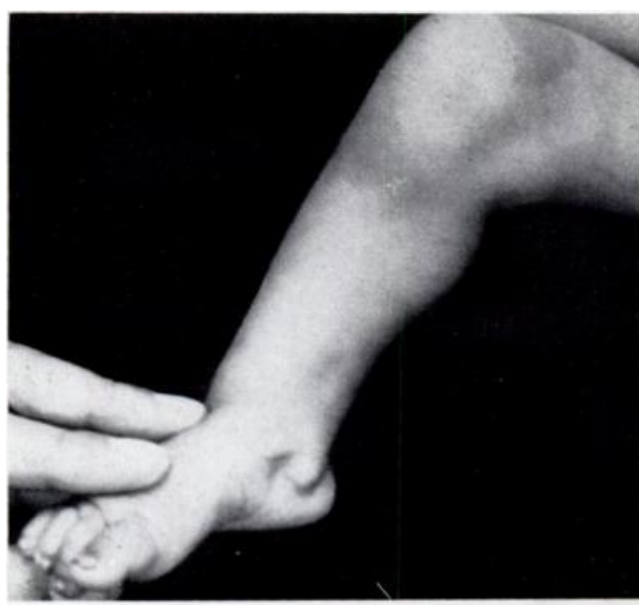

FIG. 1

FIGS. 1 AND 2

Case 1-The clinical appearance and radiographs of the right leg before operation.

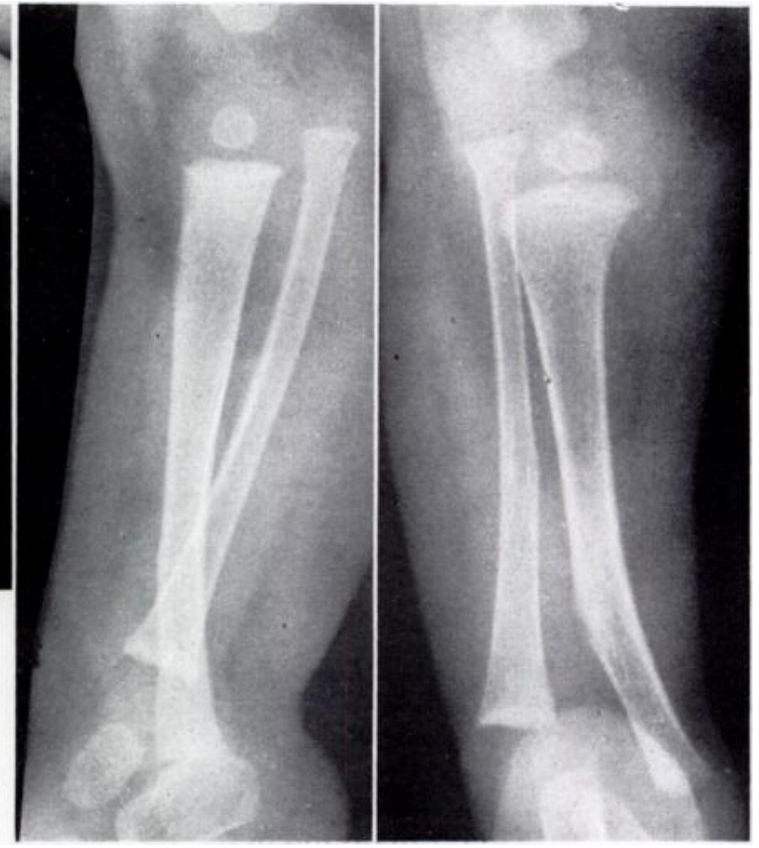

Fig. 2

centimetres shorter than the left. The upper end of the right fibula was felt at a higher level than the tibia and was freely mobile. There was an irregular café-au-lait patch just above the front of the right knee. There were no neurological or muscular defects nor were any other congenital anomalies detected. Radiographs showed displacement of the lower end of the tibia from the ankle mortise. The tibia was deviated medially and tapered towards its lower end (Fig. 2). There was diastasis of the lower ends of the tibia and fibula. At the knee 


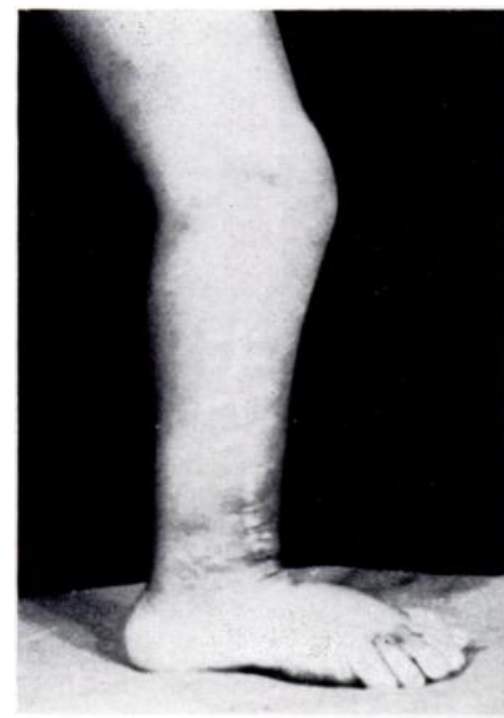

FiG. 3

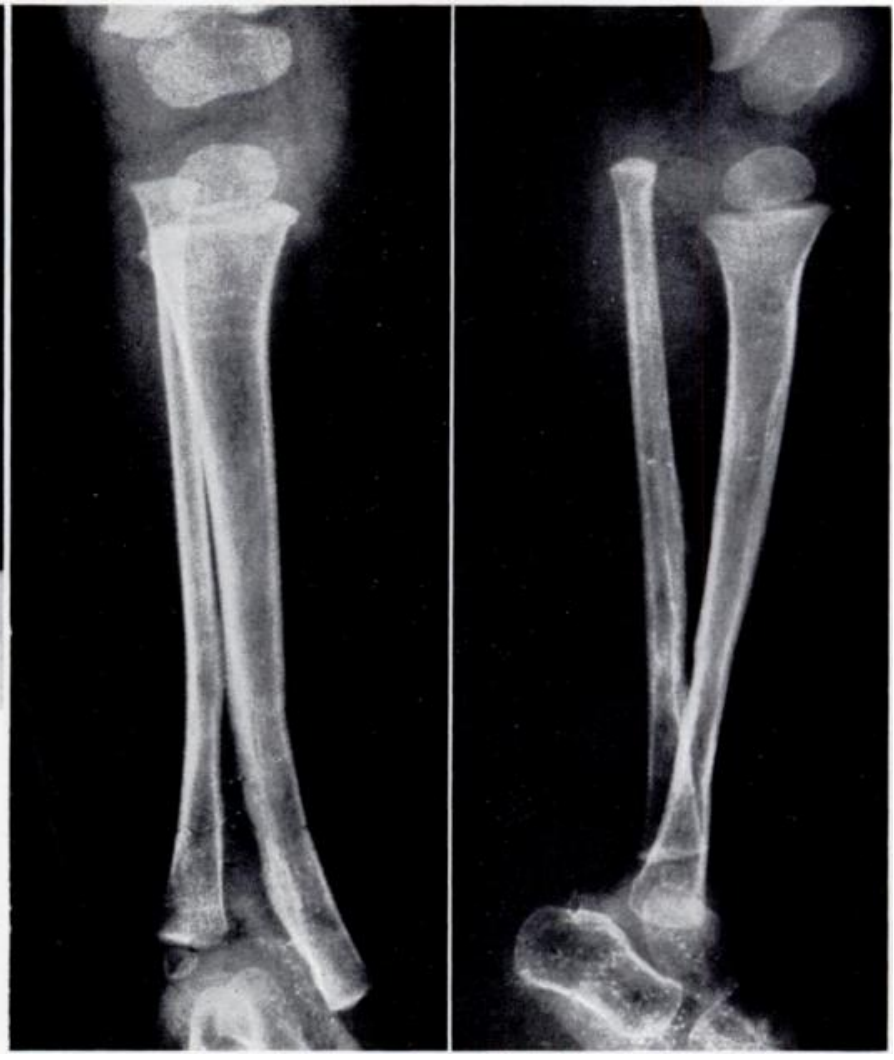

FIG. 4

the fibula was displaced markedly upwards relative to the tibia. An earlier antero-posterior radiograph taken at the age of one month, which was brought by the parents, showed the same anomalies.

Before operation in March 1970, when the child was ten months old, further radiographs revealed little change in the deformities, but the upper tibial epiphysis had appeared.

Operation-The right ankle and the lower half of the tibia and fibula were exposed through an antero-lateral incision. It was observed that the lower end of the fibula was placed anteriorly in relation to the talus, and the articular end of the tibia was subluxated posteriorly as well as deviating medially and tapering towards its lower end. The outer part of the distal articular surface of the tibia was absent. As a result the talus did not articulate with the tibia. The lower ends of the tibia and fibula were mobilised, brought together, and held in position by strong circumferential sutures of chromic catgut so as to form an ankle mortise. A Kirschner wire was passed through the calcaneus and talus into the tibia to maintain the alignment of the foot with the leg. The wound was closed and the limb was held plantigrade in an above-knee plaster. The Kirschner wire was removed at the end of four weeks and plaster immobilisation was continued for a total of twelve weeks after the operation; thereafter an above-knee caliper was worn.

Progress-The foot maintained its plantigrade position (Fig. 3), and radiographs (Fig. 4) have shown progressive remodelling of the tibia with improvement in its medial angulation and in the diastasis, though some postero-inferior subluxation has persisted. The distal fibular epiphysis appeared at the age of one year, but the distal tibial epiphysis had still not yet appeared on the right side at the age of twenty months, though it has already appeared on the left. The upper end of the right fibula has remained subluxated. The child is beginning to walk in the caliper. At present there is shortening of two centimetres in the right leg. 
Case 2 (B. P. V.'s case)-A girl aged three was seen in June 1970 with congenital club foot on the right side. She was the first child in the family and was born normally to apparently healthy parents. There was no history of any congenital anomaly in the family. The child had received no previous treatment. Physical examination revealed a marked equinovarus deformity of the right foot with a prominent lateral malleolus situated over the dorso-lateral aspect of the foot, and widening of the tibio-fibular mortise. The right leg was two centimetres short. There were no neurological or muscular defects, nor was any other congenital anomaly found. Radiographs showed postero-medial deviation of the distal half of the tibia, with diastasis between the lower ends of tibia and fibula (Fig. 5). The lower epiphyses of the tibia and fibula had not yet appeared. The distal articular end of the tibia showed defective remodelling and was tapered towards the medial side. It appeared as if there was aplasia of

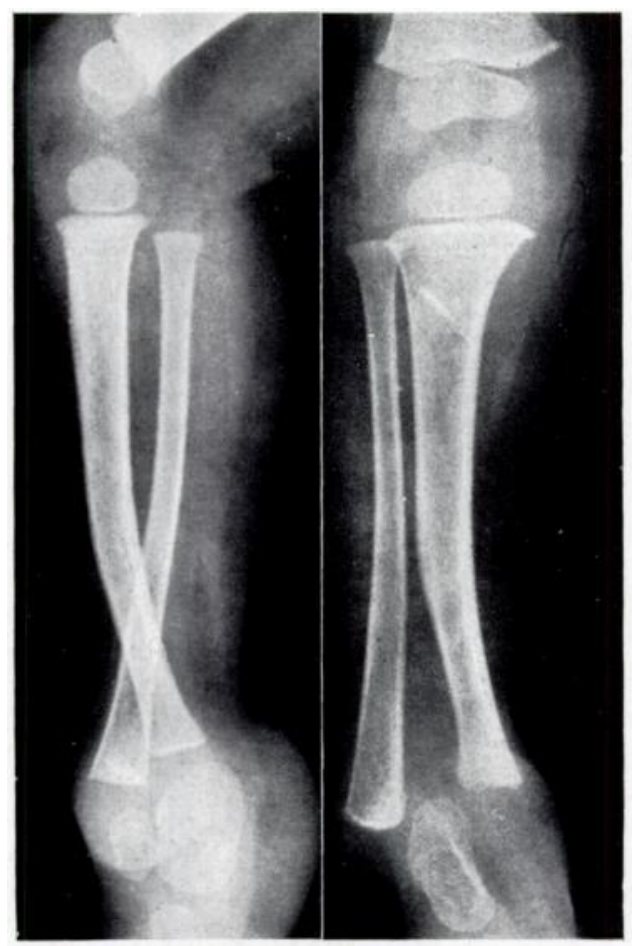

Fig. 5

Case 2-Radiographs before operation.

the lateral half of the distal articular end of the tibia. Among the tarsal bones the posterior part of the talus had not developed, and the centre for the navicular had not yet appeared. Operation-In August 1970 the right ankle and the lower half of the leg were exposed through an antero-lateral incision. The distal articular end of the tibia was deviated postero-medially and was not articulating with the talus. The fibula was found over the dorso-lateral aspect of the foot. The weight-bearing articular surface of the tibia was deficient. The lower ends of tibia and fibula were mobilised, the two bones brought together and fixed by a transverse screw to reconstitute the tibio-fibular mortise. The calcaneal tendon was lengthened to give a plantigrade foot. Because of the severe posterior displacement of the distal end of the tibia, the talus could not be brought fully within the reconstructed ankle mortise. The wound was closed and the foot was held plantigrade in an above-knee plaster. The stitches were removed after two weeks and the immobilisation was continued for twelve weeks, after which double below-knee steels were prescribed. 


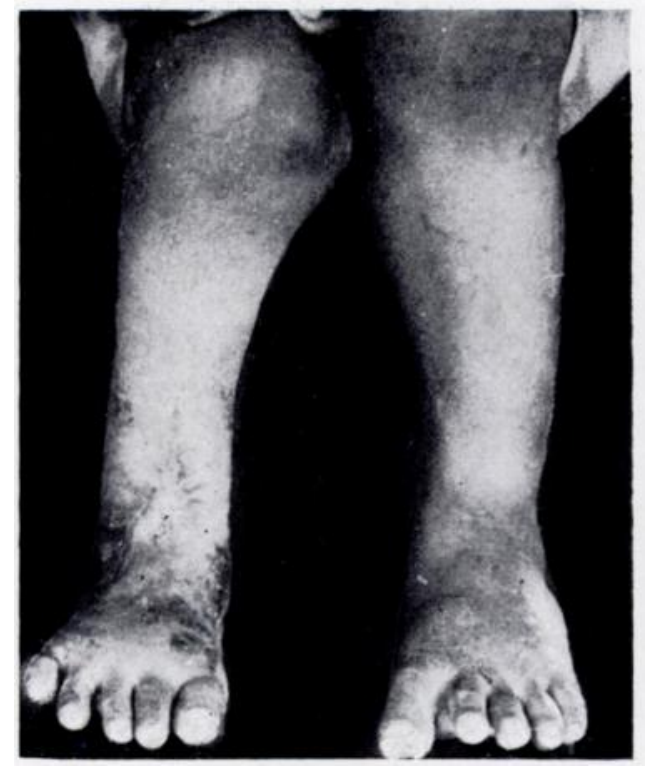

FiG. 6

Case 2-The clinical appearance after operation.

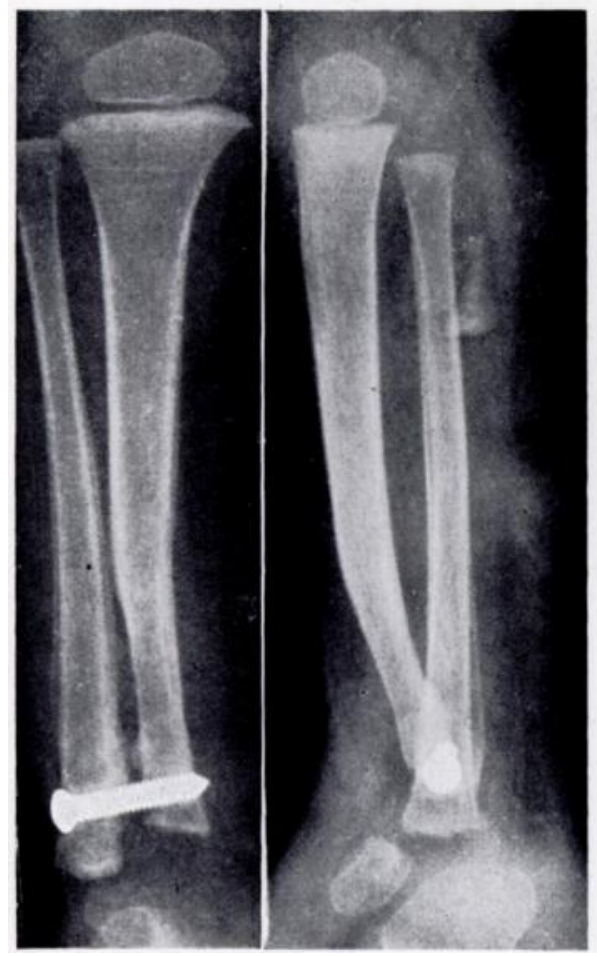

Fig. 7

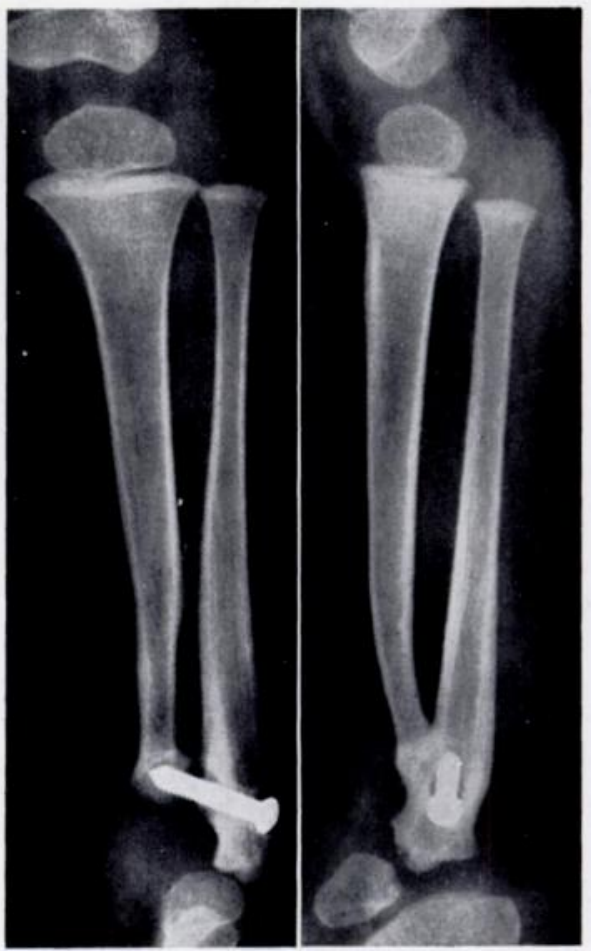

FIG. 8

Case 2-Radiographs soon after operation (Fig. 7) and six months later (Fig. 8). 
Progress-At follow-up in March 1971 the foot had maintained the plantigrade position (Fig. 6) and the child was walking in the brace. Radiographs (Figs. 7 and 8) showed a progressive improvement in the medial angulation of the tibia with a satisfactory tibio-fibular mortise, but the posterior angulation persisted. The distal epiphysis of the tibia and fibula did not appear until the age of three years and nine months and the longitudinal growth of the lower end of the tibia lagged behind that of the fibula (Fig. 8). The posterior part of talus had not developed and the ossific nucleus of the navicular bone had just appeared. The right leg was short by two and a half centimetres.

\section{DISCUSSION}

Both these children presented with an apparent equinovarus deformity of the right foot. Radiographs in both revealed diastasis of the inferior tibio-fibular mortise with medial deviation of the distal half of the tibia. In Case 1 the whole tibia was displaced posteroinferiorly and the upper end of the fibula was unduly mobile. At operation the distal articular end of tibia was found deficient in both the cases. The non-appearance of the distal tibial epiphysis in both children implies that the primary abnormality might be an osteochondrosis of the distal tibial epiphysis. Hassler, Heyman and Bennett (1960) reported two cases of osteochondrosis of the distal tibial epiphysis in which the distal tibial epiphyses appeared late and subsequently fragmented, but in neither was there any diastasis. In the cases described here it appears that the primary abnormality of the distal tibial epiphysis led to non-development of the distal articular end of the tibia and consequent tibio-fibular diastasis. Case 2, in addition, showed abnormal ossification of the lower fibular epiphysis, the posterior part of the talus and the navicular. The lower end of the fibula was situated over the dorso-lateral aspect of the foot in both the cases. This condition is probably akin to a localised epiphysial growth disturbance such as those in the distal radial epiphysis (Madelung's deformity), proximal tibial epiphysis (Blount's disease), and the proximal femoral epiphysis (infantile coxa vara). In our cases apparently no genetic or hereditary factors were concerned. Both were girls, both a first child and both had the right leg affected. Probably this is coincidental.

With the meagre experience and no long-term follow-up, it would be unjustified to comment about the treatment and prognosis of this condition. An attempt has, however, been made in both the cases to restore a plantigrade foot and reconstruct the tibio-fibular mortise. Subsequent follow-up will show the growth behaviour of the distal tibial epiphysis.

\section{SUMMARY}

1. Two cases of congenital diastasis of the inferior tibio-fibular mortise are described.

2. No previous description of this condition has been found in the literature.

3. It is suggested that the cause is osteochondrosis of the distal tibial epiphysis associated with a club foot.

\section{REFERENCE}

Hassler, W. L., Heyman, C. H., and Bennett, G. W. (1960): Osteochondrosis of the Distal Tibial Epiphysis. Journal of Bone and Joint Surgery, 42-A, 1261. 